\title{
Near real-time selection of high redshift GRBs with Swift
}

\author{
S. Campana ${ }^{1}$, G. Tagliaferri ${ }^{1}$, D. Malesani ${ }^{1}$, L. Stella ${ }^{1}$, P. D’Avanzo ${ }^{1,4}$, G. Chincarini ${ }^{1,5}$, and S. Covino ${ }^{1}$ \\ 1 INAF - Osservatorio Astronomico di Brera, via Bianchi 46, 23807 Merate (Lc), Italy \\ e-mail: campana@merate.mi .astro.it \\ 2 International School for Advanced Studies (SISSA/ISAS), via Beirut 2-4, 34016 Trieste, Italy \\ 3 INAF - Osservatorio Astronomico di Roma, via Frascati 33, 00040 Monteporzio Catone (Roma), Italy \\ 4 Università degli Studi dell'Insubria, Dipartimento di Fisica e Matematica, via Valleggio 11, 22100 Como, Italy \\ 5 University of Milano Bicocca, Department of Physics, Piazza delle Scienze 3, 20126 Milano, Italy
}

Received 18 October 2006 / Accepted 12 January 2007

\section{ABSTRACT}

\begin{abstract}
A good fraction of GRBs detected by Swift have a high redshift (up to $z=6.3$, so far). Their study allows us to investigate, among other things, cosmic star formation in the early Universe (possibly up to the re-ionization era) and the chemical enrichment of the high-redshift gas. Here we present and discuss a selection procedure that identifies high-redshift $(z \gtrsim 5)$ candidates based only upon the promptly-available information provided by Swift. This method relies upon Galactic extinction, GRB duration, and the absence of an optical counterpart in the UVOT telescope onboard Swift. This tool may provide an extremely effective way to locate high-redshift astrophysical objects and to follow them in the optical/NIR band in near real time.
\end{abstract}

Key words. gamma rays: bursts - cosmology: early Universe

\section{Introduction}

The study of GRBs at high redshift holds great promise by allowing for the investigation of GRB environments and exploration of both galaxy formation and chemical evolution up to the very young Universe.

In almost two years of operations, Swift has revolutionized the fast-response multi-wavelength studies of gamma-ray bursts (GRBs). On average, the Burst Alert Telescope (BAT, 15$350 \mathrm{keV}$, Barthelmy et al. 2005) localizes 2-3 GRBs per week (2-3' precision at gamma-ray energies); the X-Ray Telescope (XRT, 0.3-10 keV, Burrows et al. 2005) localizes $~ 70 \%$ of them in $<200 \mathrm{~s}$ down to an accuracy of 3-5" (Moretti et al. 2006). At the same time, the Ultraviolet/Optical Telescope (UVOT, 170$600 \mathrm{~nm}$, Roming et al. 2005) looks for optical counterparts. The GRB sample detected by Swift has, on average, significantly dimmer optical afterglows than the GRB sample of BeppoSAX and HETE-2. In particular, about $1 / 3$ have no optical counterpart, despite deep and prompt searches. Indeed, some of these "dark bursts" have relatively bright near-infrared (NIR) counterparts, implying that their afterglows are not just intrinsically faint but that they also suffer substantial obscuration (either from dust or neutral hydrogen) or alternatively are at high redshift. The mean redshift of the Swift bursts is $\langle z\rangle=2.8$, significantly higher than what has been measured for pre-Swift events (Jakobsson et al. 2006). Population synthesis models suggest that $\gtrsim 7-10 \%$ of the detected GRBs are at $z>5$ (Bromm \& Loeb 2006; Jakobsson et al. 2006; Daigne et al. 2006). Given the possible preference of GRBs for low-metallicity environments, the fraction at high redshift may be even higher (Natarajan et al. 2005).

These findings support the possibility that a significant fraction of the optically dark GRBs detected by Swift are at a very high redshift. GRB 050904 at $z=6.29$ (Kawai et al. 2006; Totani et al. 2006; Tagliaferri et al. 2005) might therefore be only the first discovered GRB of a population of very high-redshift events. There are already five GRBs at $z \gtrsim 5$. There might well be GRBs at a comparable or even higher redshift in the present Swift sample, the optical/NIR afterglow of which could not be detected due to observational difficulties involved in identifying of the afterglows and in acquiring their spectra. For example, a possibly very high redshift event was GRB 060116, for which we derived a best-fit photometric redshift of $z_{\text {phot }}=6.7$, with a less probable solution at $z_{\text {phot }}=4.1$ (Grazian et al. 2006). An attempt to derive a spectrum was unsuccessful, because the source was too weak due to the long delay ( 2.7 d) between the GRB and the observation (Fernández-Soto et al. 2006, in preparation). This testifies to the importance of a fast reaction.

Here we describe a simple and fast selection procedure able to spot high-redshift candidate GRBs. This is based on a combination of the GRB duration and the lack of an optical counterpart in the early UVOT data in low-extinction locations in our Galaxy.

\section{Selection procedure}

We start from the Swift sample of GRBs with known redshift (45 as of 30 Sep. 2006). The distribution of redshift-corrected $T_{90}$ (i.e. the time containing the $90 \%$ of the GRB fluence in the 15-350 keV BAT energy range) is shown in Fig. 1. It is clearly apparent that long GRBs are relatively rare (about $11 \pm 7 \%$ of all GRBs, including the underluminous ones, have an intrinsic $\left.T_{90}=T_{90}^{i} /(1+z)>60 \mathrm{~s}\right)$. However, the redshift increases the observed $T_{90}$. Taking the intrinsic rest-frame distribution of $T_{90}^{i}$ at $z=5$, the fraction of observed GRBs with $T_{90}^{i}(@ z=5)>60 \mathrm{~s}$ would be $56 \pm 19 \%$. By considering long GRBs with (observed) $T_{90}>60 \mathrm{~s}$, we can exclude a large fraction of GRBs in the redshift interval 1-3 (see Fig. 2) and select $31 \pm 8 \%$ of the GRB 


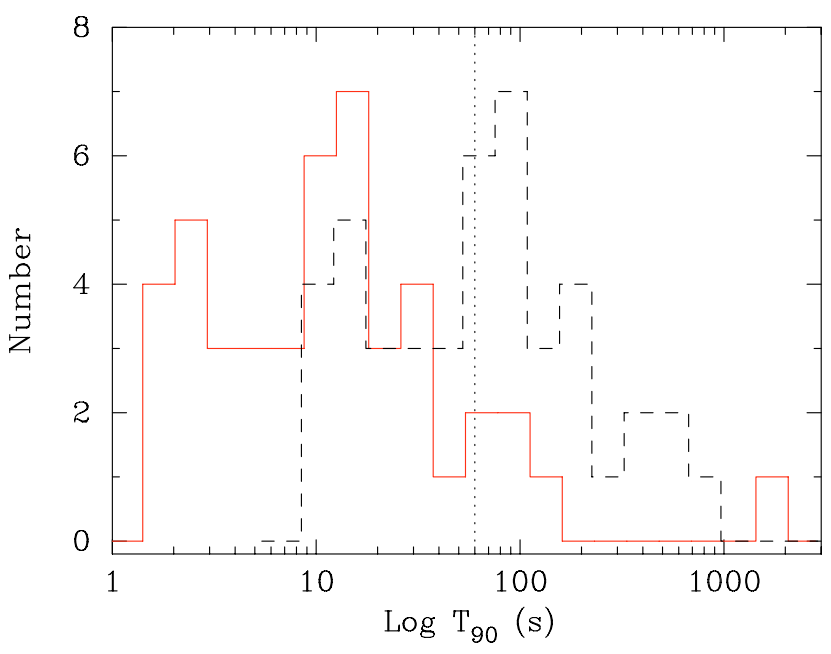

Fig. 1. Intrinsic distribution of $T_{90}^{i}$ durations observed by Swift-BAT corrected for the cosmological time dilation (redshift). The dashed-line histogram shows the observed distribution $\left(T_{90}^{i}\right)$, the continuous-line histogram shows the same distribution shifted at $z=5$. The vertical dotted line marks our reference constraint $T_{90}>60 \mathrm{~s}$.

sample with known redshift and $30 \pm 7 \%$ of the full Swift sample (160 GRBs). The $T_{90}>60 \mathrm{~s}$ threshold is somewhat empirical. Considering the intrinsic distribution of $T_{90}$ and their redshift distribution, we compute that about $12 \%$ of GRBs with $T_{90}>$ $60 \mathrm{~s}$ have $z>5$. The percentage slowly changes to $10 \%$ or $13 \%$ for a threshold at $40 \mathrm{~s}$ or $80 \mathrm{~s}$. However, given the relatively small number of GRBs we find a saturation of this fraction starting around $60-80 \mathrm{~s}$, leading us to set our threshold to $60 \mathrm{~s}$.

This is clearly not enough to identify high redshift GRBs. We add the lack of an UVOT counterpart in the first short (a few tens of seconds) $V$ image to this cut. In order not to select GRBs that are heavily absorbed in our Galaxy, we further request that the Galactic $E(B-V)<0.1$. UVOT observes shortward of $5500 \AA$ and is virtually blind to objects at $z \gtrsim 5$ due to Lyman drop out. Given that GRBs were all observed within a few hundred seconds and because UVOT observing strategy is the same for all the bursts, the lack of a UVOT counterpart is equivalent to the request that $V>19-21$. The rationale behind this cut is to select highly absorbed or high-redshift GRBs. The UVOT observing strategy described above (first short $V$ image and then $100 \mathrm{~s}$ white image) has been implemented and used systematically since March 2006. Since then, 43 GRBs have been detected by Swift. Of these 14 were discarded due to too high a Galactic extinction $(E(B-V)>0.1)$. Of the $29 \mathrm{GRBs}$ left, 17 do have an optical counterpart detected by UVOT $(59 \pm 25 \%)$.

The intersection of the two constraints is extremely powerful. In the sample of 29 GRBs we have 12 GRBs without a prompt UVOT counterpart and only 4 with $T_{90}>60 \mathrm{~s}$ (see Fig. 3). These are GRB 060904A, GRB 060814, GRB 060522, and GRB 060510B. The last two GRBs have a spectroscopic redshift of $z=5.11$ and $z=4.9$, respectively (Cenko et al. 2006; Price 2006). Actually, GRB 060522 was detected by UVOT in the $100 \mathrm{~s}$ white-light image at $19.65 \pm 0.21$ but not in the $400 \mathrm{~s}$ $V$ band exposure starting at about $140 \mathrm{~s}$ after burst trigger with an upper limit of $V>20.1$ (Fox et al. 2006; Holland 2006). GRB 060904A was not detected $3.9 \mathrm{~h}$ after the burst down to $R>22.0$ (Cenko \& Rau 2006). In the NIR, Subaru observations did not detect the afterglow down to $J>21.0,5 \mathrm{hr}$ after the burst (Aoki et al. 2006). The $K$-band afterglow of GRB 060814 was detected at $K \sim 18,4.4 \mathrm{~h}$ after the burst (Levan et al. 2006).

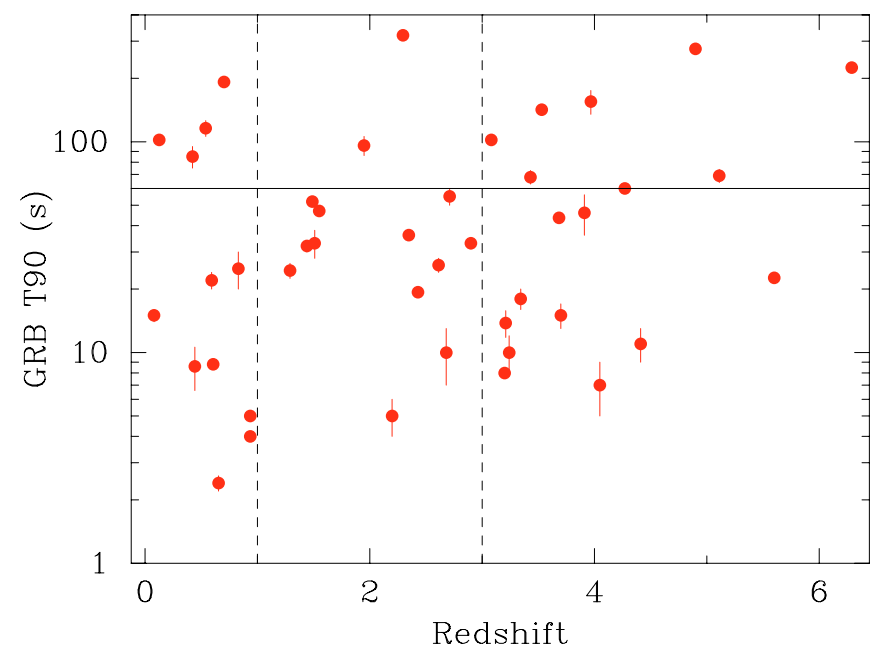

Fig. 2. Redshift distribution of the complete sample of 45 Swift GRBs versus their $T_{90}$, i.e. the time containing $90 \%$ of the BAT $15-350 \mathrm{keV}$ fluence. The horizontal line marks our selection criteria for long-distant GRBs, and the two vertical dashed lines indicate the selected redshift interval. Basically, by requiring $T_{90}>60 \mathrm{~s}$ we mainly select closeby GRBs with long durations (like GRB 060218 or GRB 060614) or distant $(z>3)$ GRBs.

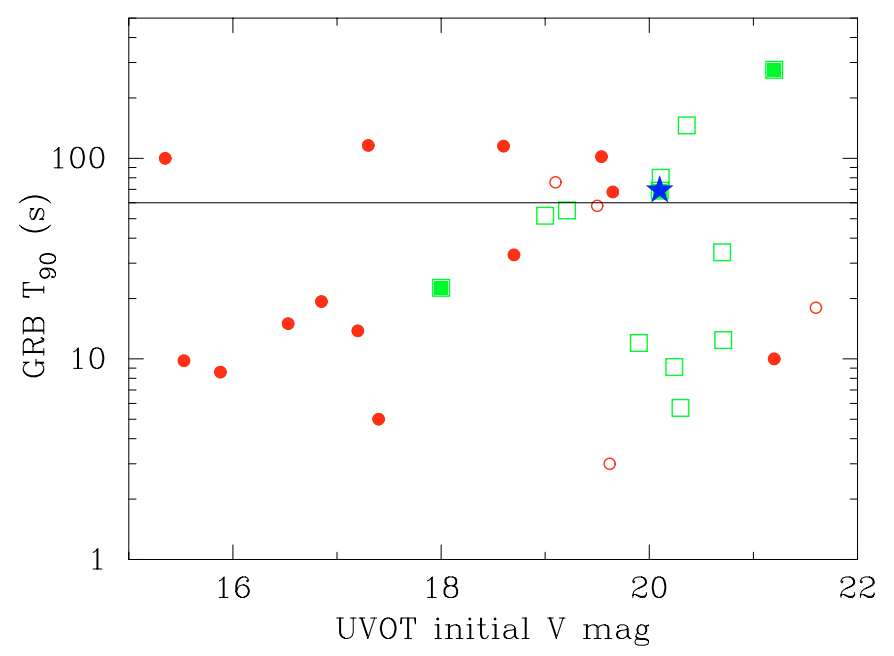

Fig. 3. Distribution of UVOT $V$ magnitudes for all Swift GRBs from March 2006 versus their $T_{90}$ duration. Circles mark GRBs with a detection with UVOT (in the $V$ band). Filled circles are GRBs with a spectroscopic redshift. Squares instead mark GRBs without a UVOT detection. Filled squares are GRBs with measured redshift. The star marks GRB 060522 with a non-detection in the $V$ band but a detection in white-light with UVOT. The combination of $T_{90}>60 \mathrm{~s}$ and absence of UVOT counterpart can be used to single out high-redshift GRBs. We have 4 objects in this space of parameters: two of them do not have a secure redshift (they have strong upper limits in the $R$ band and one has a $K$ band detection), and a spectroscopic redshift with $z \gtrsim 5$ has been measured for two of them. We note that GRB 050904 at $z=6.29\left(T_{90}=225 \pm 10 \mathrm{~s}\right.$, no optical counterpart with $V>20.1$, and $E(B-V)=0.06)$ satisfies all our selection criteria, but being observed before March 2006 is not included in the present sample.

The VLT observations taken 58 min after the event showed an $R \sim 24$ object consistent with the afterglow, as well as other closeby sources. The night was not photometric and the same object was $R=23.61 \pm 0.08$ the next day. ISIS photometry did not detect this object as variable, so we conclude that it is the likely host galaxy. These deep non-detections (or high magnitudes) in 
the $R$ band and observations in the NIR suggest the presence of intrinsically heavily absorbed afterglows or high-redshift afterglows.

\section{Conclusions}

We have presented a powerful method for singling out highredshift GRBs based on the promptly available data from Swift: GRB time duration $\left(T_{90}>60 \mathrm{~s}\right)$, lack of an optical counterpart in the UVOT data, and low Galactic extinction $(E(B-V)<0.1)$. These three constraints effectively select high-redshift GRBs. Basically, the first constraint preferentially selects distant GRBs whose duration is stretched by a cosmological time dilation. The second constraint picks up highly extincted or high-redshift objects (UVOT observes shortward of $5500 \AA$, becoming blind at $z \gtrsim 5$ due to Lyman drop out). A further constraint on the Galactic extinction cleans the sample of heavily absorbed GRBs from our Galaxy. In a sample of $29 \mathrm{GRBs}$, we are able to isolate four events. Two of them are at a known redshift of $\sim 5$ (GRB 060522 at $z=5.1$ and $060510 \mathrm{~B}$ at $z=4.9$ ). Of the remaining two, one has a large color index $R-K \gtrsim 5.6$ a few hours after the event and the other has firm upper limits in $R>22$ and in $J>21$ (about $5 \mathrm{~h}$ after the burst). These observations indicate that two out of four GRBs are high-redshift objects and another one is consistent with being a high-redshift object. If GRB 060814 has a relatively bright host galaxy it should instead be a highly reddened object. Our selection procedure is effective at selecting confirmed high-redshift GRBs $(\gtrsim 50 \%)$ but at variance with other selection techniques is characterized by a low contamination. This is very important because it will allow us to immediately identify the few objects that deserve the fastest spectroscopy with an 8-m class telescope.

We stress that our selection criteria can be applied based on the prompt information provided by Swift within a few hours (the computation of the $T_{90}$ contained in the refined BAT circular the main limitation). Additional criteria might be an X-ray column density consistent with the Galactic value since the instrinsic column density is reduced roughly by $\sim(1+z)^{2.6}$ (Campana et al. 2006; even if for GRB 050904 we find a larger column density with respect to the Galactic value, see Cusumano et al. 2006). Another criterion is a relatively smooth prompt (BAT) light curve, since GRB spikes at high redshift are dilated by cosmological redshift.
High-redshift GRBs with a prompt follow-up might yield a wealth of unprecedented information on the chemical environment of high-redshift star formation. In principle the metallicities (through $\mathrm{Fe}$ and $\mathrm{Si}$ column densities), dust depletion ( $\mathrm{Zn}$ abundances), the gas density, and the location with respect to the GRB (through observation of fine-structure excited states) can be traced. Some of these measurements have already been done with the spectroscopy of GRB 050904, which allowed a lower limit to be derived on the metallicity for $\mathrm{Si}, \mathrm{S}, \mathrm{C}, \mathrm{O}$. The gas density and location were evaluated with $\mathrm{Si}$ fine structure lines (Kawai et al. 2006). In addition, the neutral fraction of IGM was also measured (Totani et al. 2006). With more objects (possibly at even higer redshift), the star-formation history and gas content of the Universe up to the re-ionization era can be probed. Our technique may provide one of the most effective ways to single out and study objects in the high-redshift Universe.

Acknowledgements. This work was supported by ASI grant I/R/039/04 and PRIN 2005025417.

\section{References}

Aoki, K., Tanaka, I., \& Kawai, N. 2006, GCN Circ., 5522

Barthelmy, S. D., Barbier, L. M., Cummings, J. R., et al. 2005, Space Sci. Rev., 120,143

Bromm, V., \& Loeb, A. 2006, ApJ, 642, 382

Burrows, D. N., Hill, J. E., Nousek, J. A., et al. 2005, Space Sci. Rev., 120, 165

Campana, S., Romano, P., Covino, S., et al. 2006, A\&A, 449, 61

Cenko, S. B., \& Rau, A. 2006, GCN Circ., 5512

Cenko, S. B., Berger, E., Djorgovski, S. G., Mahabal, A. A., \& Fox, D. B. 2006, GCN Circ., 5155

Cusumano, G., Mangano, V., Chincarini, G., et al. 2006, Nature, 440, 164

Daigne, F., Rossi, E. M., \& Mochkovitch, R. 2006, MNRAS, 372, 1034

Fox, D., et al. 2006, GCN Circ., 5150

Grazian, A., Fernandez-Soto, A., Testa, V., et al. 2006, GCN Circ., 4545

Holland, S. T. 2006, GCN Circ., 5158

Jakobsson, P., Levan, A., Fynbo, J. P. U., et al. 2006, A\&A, 447, 897

Kawai N., Kosugi, G., Aoki, K., et al. 2006, Nature, 440, 184

Levan, A. J., Tanvir, N. R., Rol, E., Fruchter, A., \& Adamson, A. 2006, GCN Circ., 5455

Malesani, D., \& Scarpa, R. 2006, GCN Circ., 5450

Moretti, A., Perri, M., Capalbi, M., et al. 2006, A\&A, 448, L9

Natarajan, P., Albanna, B., Hjorth, J., et al. 2005, MNRAS, 364, L8

Price, P. A. 2006, GCN Circ., 5104

Roming, P. W. A., Kennedy, T. E., Mason, K. O., et al. 2005, Space Sci. Rev., 120,95

Tagliaferri, G., Antonelli, L. A., Chincarini, G., et al. 2005, A\&A, 443, L1

Totani, T., Kawai, N., Kosugi, G., et al. 2006, PASJ, 58, 485 